\title{
Loosening and Sliding Behaviour of Bolt-Nut Fastener under Transverse Loading
}

\author{
Toshio HATTORI 1, , Minoru YAMASHITA 1, Hiroki MIZUNO 1 and Tomohiro \\ NARUSE 2 \\ 1Department of Mechanical and Systems Engineering, Gifu University, \\ 1-1 Yanagido, Gifu, 501-1193, Japan \\ 2 Mechanical Engineering Research Laboratory, Hitachi Ltd.,832-2, Horiguchi, \\ Hitachinaka, Ibaraki, 312-0034, Japan
}

\begin{abstract}
The thread joint has been frequently used for the efficient productivity and maintainability as a machine element. However, many troubles such as loosening of bolted joints or fatigue failure of bolt were often experienced. Many attentions must be paid on the improvement of the strength and the reliability of the thread joints $[1,2]$. It is generally said that the fastening axial force rapidly decreases by the rotation loosening of nuts if the relative slippage on the interfaces between nuts and fastened body goes beyond a certain critical limit [3]. This critical relative slippage $\left(\mathrm{S}_{\mathrm{cr}}\right)$ that prescribes the upper limit for preventing the loosening behaviour has been estimated according to the theoretically obtained equation considering the bending deformation of bolt and the geometrical constraint condition. In this paper, firstly we present the equation for estimating the $\mathrm{S}_{\mathrm{cr}}$ based on the fundamental cantilever deformation model. Then we present the investigated results of the deformation behaviour of bolt-nut joint under transverse loading condition considering the reaction moment by nut $\left(M_{n}\right)$. Finaly we can confirmed that these estimated results of critical relative slippage coincided well with the experimental results[4,5].
\end{abstract}

\section{Introduction}

Most of machines and products have various joint portions (e.g. fastening, welding and adhesive joints) for the effective productivity and maintainability. And bolt-nut joint, one of the joint structures is widely used as it's easiness to install and remove, produces big fastening power with small force and low price in production. However, several troubles on their strength and reliability occurred at these portions as illustrated in Fig. 1. So, serious attention must be paid to improve the strength and reliability of these portions. External load acting on the bolt-nut joint comes in two types. First, if the line of action of the forces on the joint is more or less parallel to the axes of the bolt, the joint is known to

This is an Open Access article distributed under the terms of the Creative Commons Attribution-Noncommercial License 3.0, which permits unrestricted use, distribution, and reproduction in any noncommercial medium, provided the original work is properly cited. 


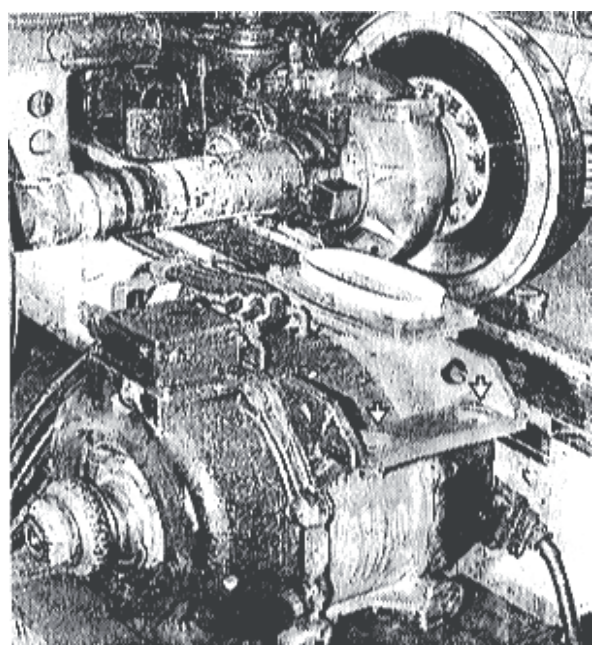

(a)

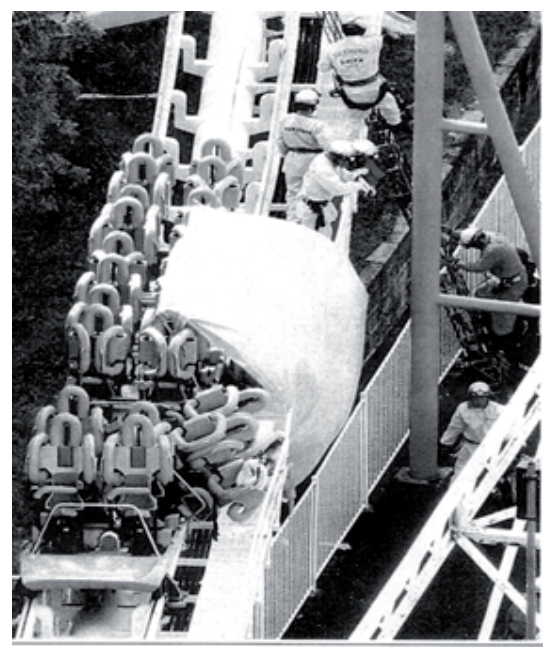

(b)

Fig. 1 Loosening and disengaged of (a) motor-fastening-bolts of 'Shinkansen' (May 1992) and (b) axle bolt of 'Steel Dragon 2000' roller coaster, Nagashima (January 2005)

be loaded in tension, $\mathrm{W}_{\mathrm{t}}$. The joint is called tensile or tension joint. Second, if the line of action of the load is more or less perpendicular to the axes of the bolt, the joint is loaded in shear, $\mathrm{W}_{\mathrm{s}}$ (also called as transverse load). It is called a shear joint. Both types are illustrated in Fig. 2. Some joints support combined tensile and transverse load and are named after the larger of the loads placed on them, be it tensile or shear. The distinction between tensile and transverse load is important, because the two types differ in the way they act to the joint, the way in which they make the joint fail, etc. According to the latest research (1), transverse load loosen to the bolt-nut joint more than tensile load. It is generally said that the fastening axial force rapidly decreases by the rotation loosening of

$\mathrm{Wt}$ $\mathrm{Wt}$

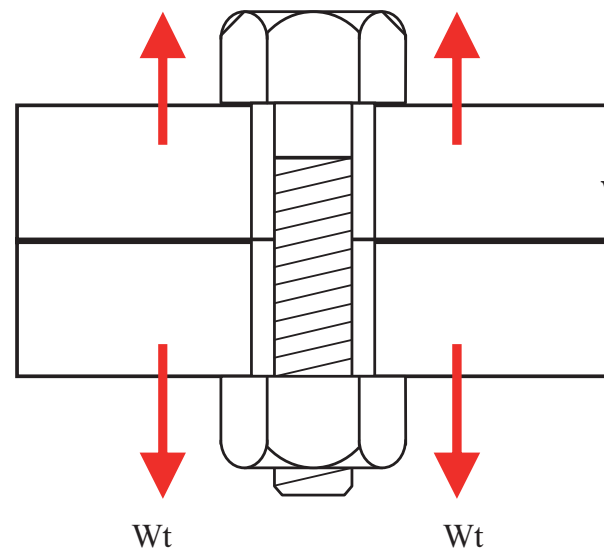

(a)

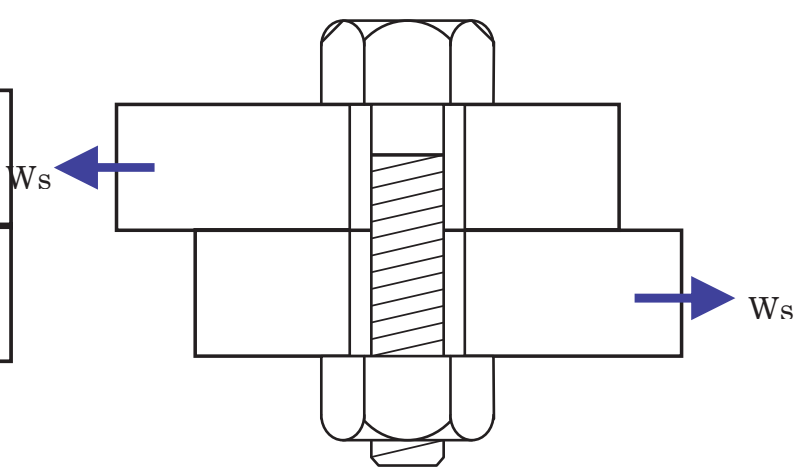

(b)

Fig. 2 Tensile joint (a) and shear joint (b) 
nuts if the relative slippage on the interfaces between nuts and fastened body goes beyond a certain critical limit [3]. This critical relative slippage $\left(\mathrm{S}_{\mathrm{cr}}\right)$ that prescribes the upper limit for preventing the loosening behaviour has been estimated according to the theoretically obtained equation considering the bending deformation of bolt and the geometrical constraint condition. In this paper, firstly we present the equation for estimating the $\left(\mathrm{S}_{\mathrm{cr}}\right)$ based on the fundamental cantilever deformation model. Then we present the investigated results of the deformation behaviour of bolt-nut joint under transverse loading condition considering the reaction moment by nut $\left(\mathrm{M}_{\mathrm{n}}\right)$. Finaly we can confirmed that these estimated results of critical relative slippage coincided well with the experimental results $[4,5]$.

\section{Mechanics of Bolt-Nut Joints}

The sliding behaviour of bolt-nut joint and reverse rotation of nut under transverse load is showed in Fig. 3. The deformation behaviour of bolt-nut joint is depends on the amount of transverse load level applied on the joint $(2,3,4)$. When the load, $\mathrm{W}$ is low, bolt and fastened components (fixed plate and movable plate) are deformed as a unit (Fig.3 (a)). No slip generated at the bolt and nut bearing surface. Then, slip generated at the contact surface between fixed plate and movable plate when $\mathrm{W}$ exceeded the frictional force (Where frictional force $=$ number of bolts, $\mathrm{n} \times$ friction coefficient, $\mu \times$ initial axial tension, $F_{b}$ ) is given to the joint. However, as shown in Fig. 3(b) when relative displacement, $\mathrm{S}$ between upper and lower plate is still small, by the bending deformation of the bolt, sliding at the bolt and nut bearing surface is not generated. Then, load and relative displacement between plates increased and at one stage (relative displacement exceeded $\Delta \mathrm{S}$ (Fig. 3(c)), slip is also occurs at the bolt and nut bearing surface. This slip leads to the reverse rotation of the nut and decrease the axial tension. The loosening behaviour of bolt-nut joint shows the same occurrence if the transverse load applied in the opposite direction. When transverse cyclic load applied to the joint, bolt axial tension slowly decreases and at the worst stage, not only loosening occurs but also the fatigue failure of the bolt. So, it is important to evaluate both loosening behaviour and fatigue failure of the bolt-nut joint

(a)

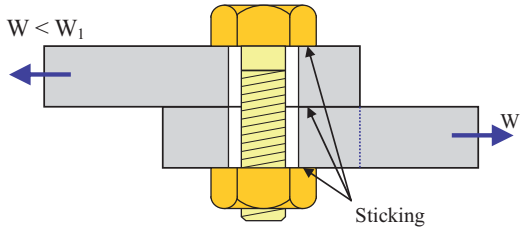

(b) $\mathrm{W}_{1}<\mathrm{W}<\mathrm{W}_{2}$

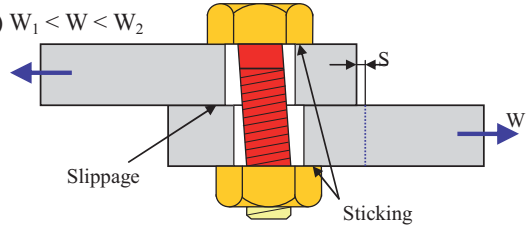

(c) $\mathrm{W}>\mathrm{W}_{2}$

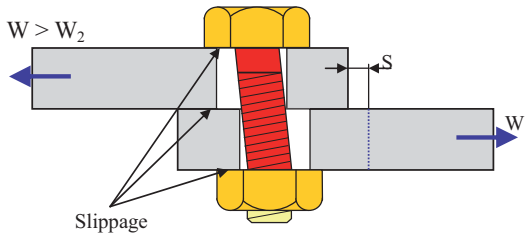

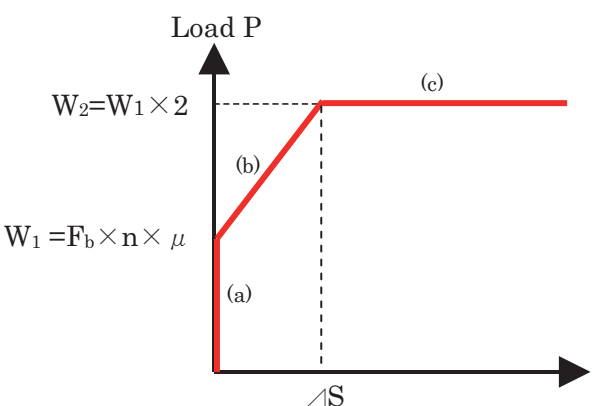

Fig. 3 Behavior of the bolt-nut joint in different load condition. 
Concerning the loosening rotation of nut under transverse loading, Yamamoto and Kasei [6] proposed the equation that evaluates the critical relative slippage, $S_{\mathrm{cr}}$. Derivation of evaluation equation is given as below.

Intended bolt and nut is shown in Fig.4. Consider that contact surface is exists between movable plate surface and nut bearing surface. Initial axial tension of bolt is set as $F_{b}$. When bolt-nut joint is subjected to shear direction external force, transverse load, $\mathrm{W}_{\mathrm{r}}$ and reaction moment, $\mathrm{M}_{\mathrm{n}}$ act at the thread portion. Here, we can calculate the bolt shank's deflection that caused by both transverse load and reaction moment by considering the bolt as a beam. Furthermore, bolt bearing surface is inclines since it is not fixed and proportional to the bending moment of bolt head. Bolt shank' $\mathrm{s}$ deflection, $\Delta \mathrm{aF}$ that caused by transverse load, $\mathrm{W}_{\mathrm{r}}$ is shown in following equation :

$$
\Delta_{a F}=W_{r}\left(\frac{l_{g}^{3}}{3 E I_{g}}+\frac{l_{p}^{3}}{3 E I_{p}}+\frac{l_{g} l_{p} l_{n}}{E I_{g}}\right)
$$

where,

$\mathrm{E}=$ Young Modulus of bolt

$\mathrm{Ig}=$ second moment of area of bolt's cylindrical portion

$\mathrm{Ip}=$ second moment of area of thread portion

Then, bolt shank' s deflection, $\Delta$ aM that caused by reaction moment, $M_{n}$ is shown as below :

$$
\Delta_{a M}=-M_{n}\left(\frac{l_{g}^{2}}{2 E I_{g}}+\frac{l_{p}^{2}}{2 E I_{p}}+\frac{l_{g} l_{p}}{E I_{g}}\right)
$$

And inclination angle of bolt head is defined as :

$$
\theta=k_{w}\left(W_{r} l_{n}-M_{n}\right)
$$

where

$\mathrm{kw}=$ bolt head's inclination compliance

$\mathrm{kw}$ indicates the easiness of bolt head to incline during transverse loading. Displacement magnitude, $\Delta \mathrm{a} \theta$ at bolt head caused by bending stress is shown in following equation:

$$
\Delta_{a \theta}=k_{w}\left(W_{r} l_{n}^{2}-M_{n} l_{n}\right)
$$

So, displacement magnitude of load point at thread portion, $\Delta \mathrm{S}$ which is sums of $\Delta \mathrm{aF}$, $\Delta \mathrm{aM}, \Delta \mathrm{a} \theta$ is shown in equation below :

$$
\begin{aligned}
& \Delta S=\Delta_{a F}+\Delta_{a M}+\Delta_{a \theta} \\
= & W_{r}\left(\frac{l_{g}^{3}}{3 E I_{g}}+\frac{l_{p}^{3}}{3 E I_{p}}+\frac{l_{g} l_{p} l_{n}}{E I_{g}}+k_{w} l_{n}^{2}\right)-M_{n}\left(\frac{l_{g}^{2}}{2 E I_{g}}+\frac{l_{p}^{2}}{2 E I_{p}}+\frac{l_{g} l_{p}}{E I_{g}}+k_{w} l_{n}\right)
\end{aligned}
$$


Coefficient friction of nut bearing surface is set as $\mu \mathrm{w}$. When transverse load, $\mathrm{W}_{\mathrm{r}}$

$$
W_{r}=\mu_{w} F_{b}
$$

reaches relative slippage at the nut bearing surface occurs and critical relative slippage, $\mathrm{S}_{\mathrm{cr}}$ is defined as below :

$$
\begin{aligned}
S_{c r} & =2 \Delta \\
& =2\left[F \mu_{w}\left(\frac{l_{g}{ }^{3}}{3 E_{b} I_{g}}+\frac{l_{p}{ }^{3}}{3 E_{b} I_{p}}+\frac{l_{g} l_{p} l_{n}}{E_{b} I_{g}}+k_{w} l_{n}{ }^{2}\right)-M_{n}\left(\frac{l_{g}{ }^{2}}{2 E_{b} I_{g}}+\frac{l_{p}{ }^{2}}{2 E_{b} I_{p}}+\frac{l_{g} l_{p}}{E_{b} I_{g}}+k_{w} l_{n}\right)\right]
\end{aligned}
$$

\section{Derivation of Nut Reaction Moment $\mathbf{M}_{\mathbf{n}}$}

Yamamoto and kase[6] present the estimation equation of reaction moment as follows by using the mechanical model as shown in Fig. 4(a).

where

$$
M_{n}=\left(\frac{m}{4}\right)\left(\frac{\mu_{b} F_{b}}{\cos ^{2} \alpha}\right)
$$

$\mu_{\mathrm{b}}=$ coefficient friction of bolt bearing surface

In this model they assumed that the contact pressures between bolt and nut are uniformly distributed on 4 corners. But the contact pressure between bolt nut will distributed on each thread, so in this paper we present new estimation equation of reaction moment using the mechanical model as shown in Fig. 4(b), as follows.

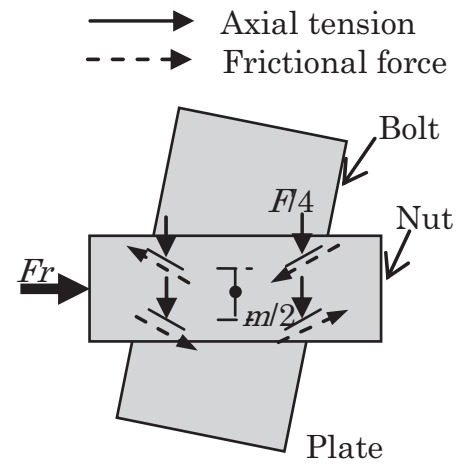

(a) Model of yamamoto and kase

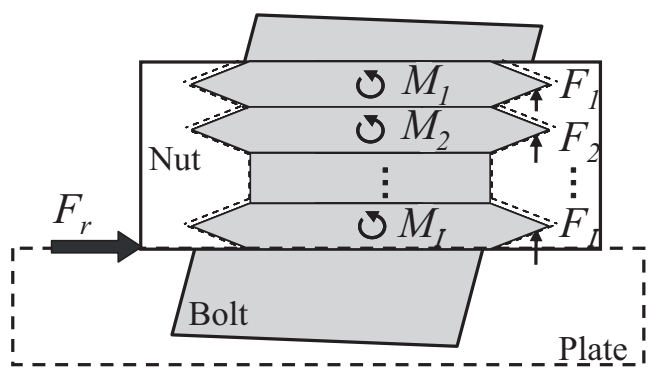

(b) Model in this study

Fig.4 Force and moment at each interface.

$$
M_{n}=\sum_{j=1}^{I} M_{j}=\frac{F_{I}}{A} Z=\frac{C F}{8} \times \frac{d^{2}+d_{1}^{2}}{d}
$$


In this equation reaction moment on each thread $\mathrm{M}_{\mathrm{j}}$ is shown as follows.

$$
M_{j}=\frac{F_{j}-F_{j-1}}{A} Z
$$

\section{Comparison with the Experimental results}

To confirm the validity of these estimation models we performed quasi-static loading tests as follows.

\subsection{Experimental methods}

Medium carbon steel plates were used for fastened components. The ordinary bolt made of carbon steel, M6, M10, M16 are used for the fastening. The mechanical properties of each bolt sizes are given in Table 1.

Bolt-nut joint and sensors are shown in Fig. 5. The fastening and bending stress of bolts are measured using strain gage mounted at the vicinity of bolt neck as shown in Fig. 6 .

Table 1 Material properties of M6, M10 and M16 bolt

\begin{tabular}{|c|c|c|c|c|c|c|}
\hline $\begin{array}{c}\text { Nominal } \\
\text { diameter }\end{array}$ & $\begin{array}{c}\text { Property } \\
\text { class }\end{array}$ & U.T.S. [MPa] & $\begin{array}{c}\text { Proof } \\
\text { stress } \\
{[\mathrm{MPa}]}\end{array}$ & Pitch & $\begin{array}{c}\text { Effective } \\
\text { stress } \\
\text { area } \\
{\left[\mathrm{mm}^{2}\right]}\end{array}$ & $\begin{array}{c}\text { Standard } \\
\text { axial } \\
\text { tension } \\
{[\mathrm{N}]}\end{array}$ \\
\hline M16 & \multirow{2}{*}{4.8} & 392 & 314 & 1.5 & 58 & 12732 \\
\hline M10 & & 39 & 1 & 20.1 & 4412 \\
\hline
\end{tabular}
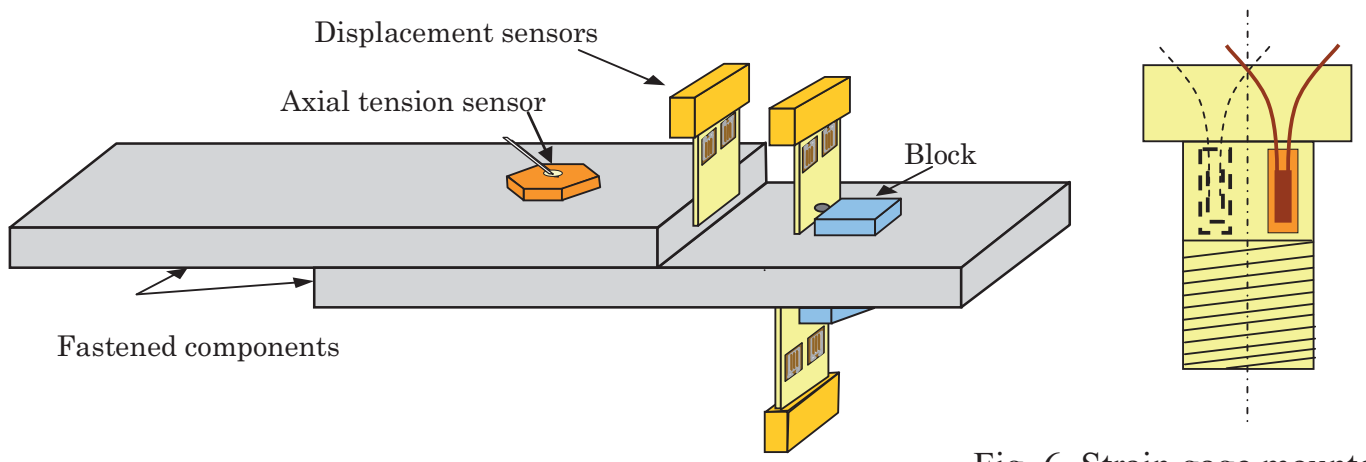

Fig. 5 Bolt-nut joints and sensors

Fig. 6 Strain gage mounted on the bolt neck

Fatigue testing machine with hydraulic pump is used in the order to generate transverse cyclic loading. Experimental apparatus is illustrated in Fig. 7.

In this experiment, static loading (tension only) is applied to the joint which is fastened by various axial tensions (depend on bolt size) and the critical load when sliding occurs between bolt bearing surface and fixed plate is examined. Transverse load, bolt axial 


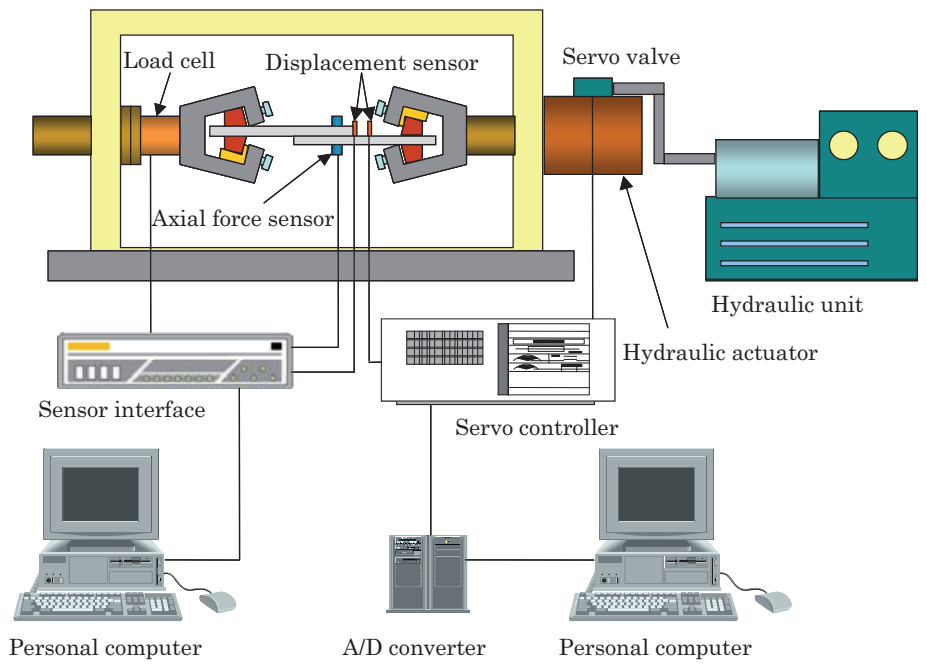

Fig. 7 Experimental apparatus

tension and relative displacement of fastened plates which is the difference displacement between fixed plate and movable plate are measured by sensors and load cell.

\subsection{Comparing results and Discussions}

Fig. 8 show the relationships between transverse load which applied to the joint vs. relative displacement between fastened plates. From these quasi static test results we can obtain the experimental critical slippage $S_{\text {cr }}$ and these values coincided well with that of obtained from the experimental loosening behaviour under repeated load cycle as shown in Fig. 9. By comparing these experimental critical slippage $S_{\mathrm{cr}}$ for each fastening force with the Eq. (7), we can obtain the experimental reaction moment $M_{n}$ as shown in Fig. 10. These experimental results coincided well with the estimated results from Eq. (9) as shown in this figure.

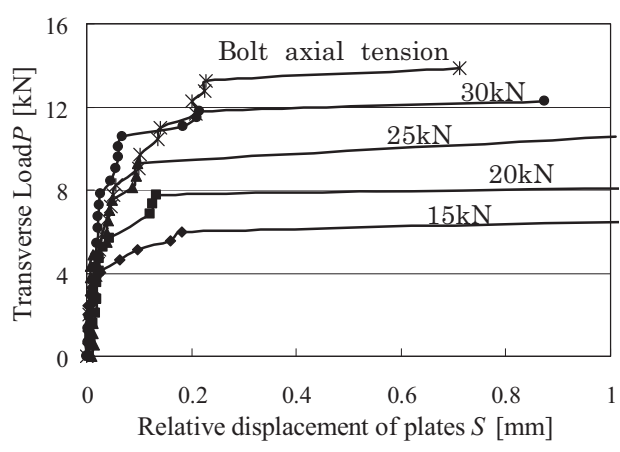

Fig.8 Dependence of transverse load on relative displacement

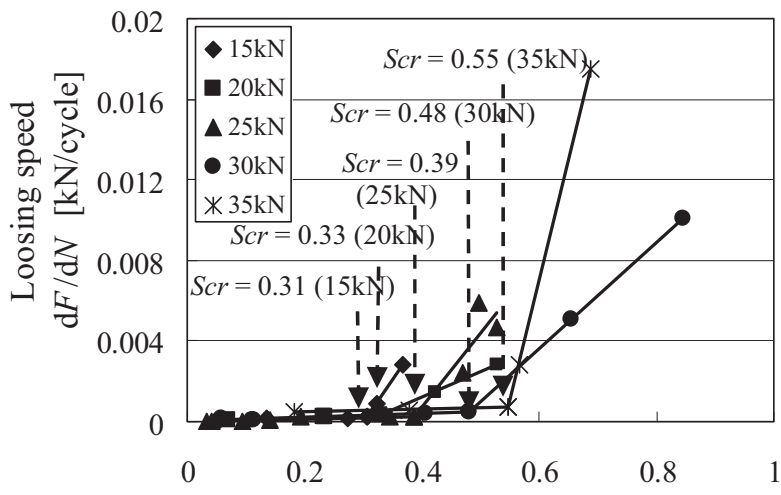

Relative displacement $S$ [mm]

Fig.9 Dependence of $\mathrm{dF} / \mathrm{dN}$ on relative displacement. 


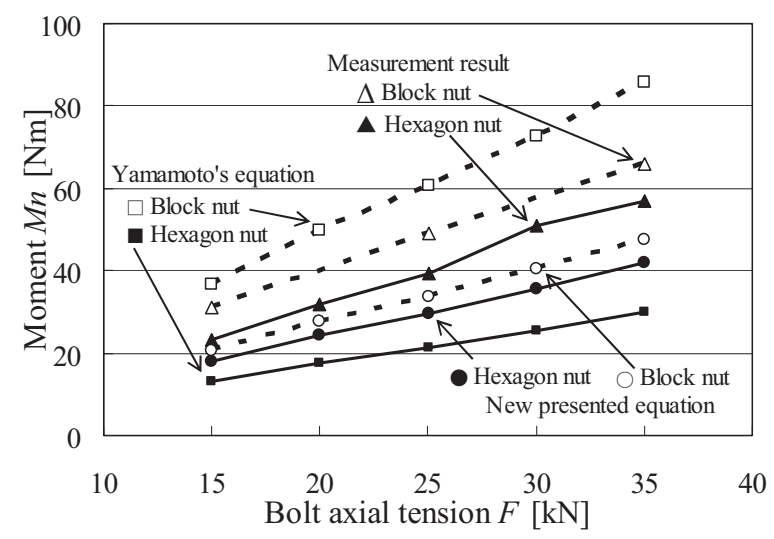

Fig.10 Relations between transverse load $P$ and reaction force moment $M_{n}$.

\section{Conclusions}

1. In this paper we introduced the estimation model of critical slippage $\mathrm{S}_{\mathrm{cr}}$ for bolt loosening under transverse loadings.

2. In this estimation method we present new prediction equation for reaction moment $\mathrm{M}_{\mathrm{n}}$ based on the bolt/nut jointing model.

3. Using these estimation methods we can predict critical slippage on each fastening conditions such as bolt sizes, bolt lengths, fastening force and so on. And these methods can be used for the CAE design tool for each machine structures.

\section{References}

1. Junker, G. H, New Criteria for Self-Loosening of Fasteners Under Vibration, SAE Transactions, Vol. 78 (1969), pp.314-335.

2. Pai, N. G. and Hess, D. P., Experimental Study of Loosening of Threaded Fasteners due to Dynamic Shear Loads: Journal of Sound and Vibration, Vol. 253, No.3 (2002), pp.585-602.

3. Izumi, S. et al., Three-dimensional Finite Element Analysis of Tightening and loosening Mechanism of Threreaded Fastener, Engineering Failure Analysis, Vol.12, No.4 (2005), pp.604-615.

4. Nakamura, M. et al., Estimation of Back-Off Loosening Limit for Bolted Joints under Transverse Cyclic Loading, Transactions of the Japanese Society of Mechanical Engineers, Series C, Vol.67, No.661 (2001), pp.2976-2980.

5. Yamamoto, A.: Principle and Design of Thread Joint, Yokendo Ltd.(1995), pp.120127.

6. Yamamoto, A. and Kasei, S., Investigations on the Self-Loosening of Threaded Fasteners under Transverse Vibration -A Solution for Self-loosening Mechanism-, Journal of the Japan Society of Precision Engineering, Vol. 43, No.4 (1977), pp.470-475. 